\title{
Visual evoked responses and visual symptoms in multiple sclerosis
}

\author{
THOMAS HOEPPNER AND FER N NDO LOLAS \\ From the Department of Neurological Sciences, Rush University, Chicago, Illinois, USA
}

SUMMARY Absolute latency, interocular difference in latency, and waveform of visual evoked responses (VER) to checkerboard reversal stimuli recorded from the midline of the skull were studied in 104 multiple sclerosis patients, 25 to 50 years of age, classified according to visual symptomatology. Group 1 had strong evidence of past or present optic neuritis. Patients with blurring of vision, diplopia, and undefined visual complaints were assigned to group 2, while group 3 contained patients with no visual symptoms but suspected diagnosis of multiple sclerosis on other grounds. The three parameters explored showed consistent association with the degree of visual involvement, as assessed by clinical impression, but their discriminatory power was diverse. Absolute latency was significantly longer in group 1 patients compared with groups 2 and 3, but it did not discriminate between the last two, whereas interocular difference in latency proved to be sensitive to differences between symptomatic (diplopia, blurring) and asymptomatic groups (2 and 3 ). Waveshapes were grouped into three categories based upon degree of distortion of the major positive peak, and their relative distribution among the three patient groups was found to be associated with symptomatology. We suggest that, in the production of symptoms such as diplopia, a temporal disparity of afferent impulses might be involved in much the same way that spatial incongruities between both eyes lead to impaired function. In this regard, interocular difference in latency rather than absolute latency would be a more accurate predictor of symptom development. The analysis of VER waveshape suggests, in addition, the importance of inhomogeneous involvement of the visual pathways in the production of symptoms during the evolution of multiple sclerosis.

The visual evoked response (VER) has become a more useful measure of visual function in recent years primarily because of the discovery that stimulation with a contrast reversing checkerboard pattern produces a very consistent response, in comparison to flash stimulation, both within and between individuals (Ciganek, 1969; Halliday and Michael, 1970; Behrman et al., 1972; Halliday et al., 1973). The reversing checkerboard pattern sliminates change in total luminance associated with flash stimulation, and the response to pattern reversal is less influenced by attention (Kopell et al., 1969). The reliability of the response, especially its latency, makes it particularly sensitive to alterations in conduction velocity in the visual pathways

Address for reprint requests: Dr Thomas Hoeppner, Department of Neurological Sciences, Rush University, 1753 W. Congress Parkway, Chicago, Illinois 60612, USA.

Accepted 27 December 1977
(Halliday et al., 1972). Thus it has gained diagnostic value in the evaluation of patients suspected of having demyelinating disease since conduction velocity changes early in the disease process and may be presumed to precede the development of symptoms (McDonald, 1974). The usefulness of the VER is enhanced by the fact that the optic nerves are among the earliest and most frequently involved sites in multiple sclerosis (Lumsden, 1970; McAlpine et al., 1972).

A number of studies have demonstrated the sensitivity of the VER latency in distinguishing normal subjects from patients with multiple sclerosis using checkerboard reversal (Halliday et al., 1973; Asselman et al., 1975) or flash (Richey et al., 1971). However, some studies suggest that the intensity or extent of visual involvement does not bear a consistent relationship to the VER (Richey et al., 1971; Halliday et al., 1973). This 
suggestion is prompted in part by the surprising observation that patients who have recovered from optic neuritis often have abnormal VER latency (Halliday et al., 1972).

In the present study we examined the intensity of visual involvement (apart from visual acuity) in patients suspected of having multiple sclerosis with regard to selected VER parameters. Our aim was to explore the notion that patients with clear evidence of optic nerve disease might have visual evoked potentials distinguishable from those of patients with other visual symptoms as well as from patients with no visual complaints. This inquiry might add insight into the mechanisms responsible for the symptoms.

\section{Methods}

Visual evoked responses were examined in 104 patients, ranging in age from 25 to 50 years, with a diagnosis of possible, probable, or definite multiple sclerosis (McAlpine et al., 1972).

Midline electrode locations on the scalp which have been found to yield a maximal response to checkerboard pattern reversal were used (Halliday et al., 1972). All responses discussed here were derived from an electrode $5 \mathrm{~cm}$ above the inion referred to electrode $\mathrm{F}_{z}$ in the 10-20 system.

The stimulus was a reversing black and white checkerboard pattern projected on a screen $1.5 \mathrm{~m}$ in front of the patient. Individual squares subtended 50 minutes of arc, and the entire pattern subtended 18 degrees horizontally by 28 degrees vertically at the eye.

Pattern reversal at $1.5 \mathrm{~Hz}$ was produced by uscillating a mirror mounted on a galvanometer using a squarewave displacement, each pattern being present for one-third of a second. The checkerboard image from a slide projector was reflected by the mirror on to a front projection screen. Patients were tested twice binocularly and twice with each eye. For each condition the average evoked response to 200 reversals was computed on a Nicolet Med 80 Computer using 256 points to represent the $350 \mathrm{~ms}$ after the stimulus.

Visual assessment included confrontation fields, pupil response, eye movements, and a funduscopic examination but patients were classified primarily on the basis of their presenting visual complaints.

Patients were divided into three groups based on their visual findings (Table 1). Group V-I $(n=35$, mean age 35.3 years) had strong evidence of optic nerve involvement. Patients in group V-II $(n=41$, mean age 34.4 years) described blurring of vision or diplopia; or less definite visual symptoms. Group V-III ( $n=28$, mean age 34.6 years) had no visual complaints but were suspected of having multiple sclerosis on other grounds.

Table 1 Classification of visual findings in patients with suspected or confirmed multiple sclerosis

\begin{tabular}{ll}
\hline Group & Visual findings \\
\hline V-I & Evidence of optic nerve involvement (demonstrable \\
scotoma, past or present optic neuritis) \\
V-II & Blurring, diplopia, and other visual symptoms \\
V-III & No visual complaints \\
\hline
\end{tabular}

\section{Results}

Figure 1 shows an example of a normal evoked response to monocular stimulation with checkerboard reversal along with average values for this laboratory. Note that the latency is much more consistent than the amplitude.

The normal difference in latency on left and right eye stimulation (interocular difference in latency) is $1.68 \pm 1.54 \mathrm{~ms}$.

Figure 2 shows the distribution of maximal VER latency for multiple sclerosis patients with different visual findings. Maximal latency is the latency of the largest positive peak between 90 and $170 \mathrm{~ms}$ (P1) for the eye having the longest latency in each patient. If two peaks were of equal height, the latency to the first was selected.

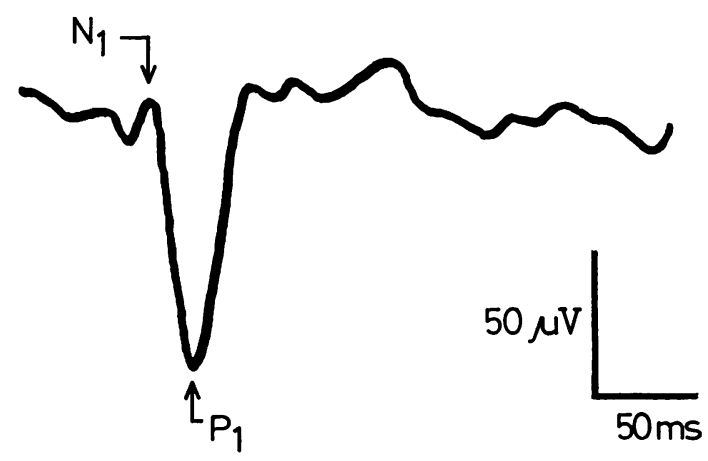

$$
\begin{aligned}
& N_{1} \text { mean latency }=68 \cdot 7 \pm 4.4 \mathrm{~ms} \\
& P_{1} \text { mean latency }=96 \cdot 4 \pm 5 \cdot 3 \mathrm{~ms} \\
& N_{1}-P_{1} \text { mean voltage }=9 \cdot 36 \pm 3.68 \mu \mathrm{VV}
\end{aligned}
$$

Fig. 1 Averaged visual evoked response values based on 16 control subjects with normal vision and no neurological complaint. Stimulus was contrast reversal of a checkerboard pattern at $1.5 \mathrm{~Hz}$. Each subject contributed an average of 400 responses. Midline electrodes $50 \mathrm{~mm}$ above the inion- $\left(F_{z}\right)$ (representative tracing, retouched). 


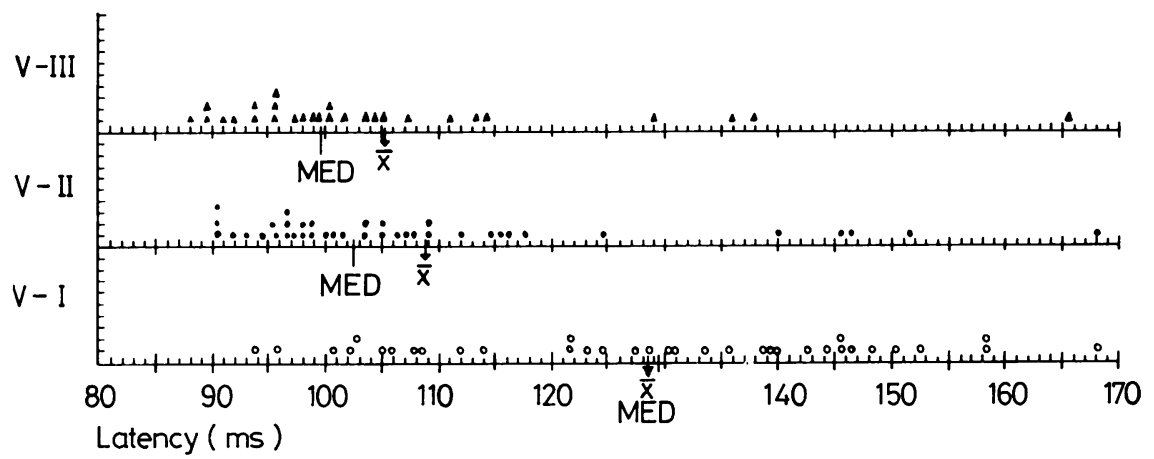

Fig. 2 VER latency of patients suspected of having, or known to have, multiple sclerosis with different visual findings.

The latency for patients in group V-I is generally much longer than that for other patients. Statistical comparisons for these data are presented in Table 2A. Patients in group V-I had the longest maximal latencies, those in group V-III the shortest, group V-II being intermediate. Differences between groups V-I and V-III and V-I and V-II were significant, while those between V-II and V-III were not.

Table 2 A Latency of the major positive peak in the visual evoked response of patients with different visual findings

\begin{tabular}{lllll}
\hline Group & Latency $(m s)$ & $t$ & $d f$ & $P$ \\
\hline V-I & $128.73 \pm 19.90$ & 4.42 & 74 & $<0.001$ \\
V-II & $108.97 \pm 18.74$ & & & \\
V-I & $128.73 \pm 19.90$ & 4.94 & 61 & $<0.001$ \\
V-III & $105.23 \pm 17.72$ & & & NS \\
V-II & $108.97 \pm 18.74$ & 0.84 & 67 & \\
V-III & $105.23 \pm 17.72$ & & & \\
\hline
\end{tabular}

\section{B Absolute interocular difference in latency (IDL)}

\begin{tabular}{lrlll}
\hline Group & \multicolumn{1}{l}{$I D L(m s)$} & $t$ & $d f$ & \multicolumn{1}{l}{$P$} \\
\hline V-I & $14.65 \pm 12.79$ & 3.31 & 73 & $<0.01$ \\
V-II & $6.23 \pm 8.20$ & & & \\
V-I & $14.65 \pm 12.79$ & 4.54 & 61 & $<0.001$ \\
V-III & $3.87 \pm 4.76$ & & & \\
V-II & $6.23 \pm 8.20$ & 3.31 & 66 & $<0.01$ \\
V-III & $3.87 \pm 4.76$ & & & \\
\hline
\end{tabular}

Interocular differences in latency discriminated between all three groups of patients including groups V-II and V-III (Table 2B). Interocular differences were greatest for group V-I and least for group V-III, correlating positively with the maximum latency measurement. Thus, while maxi- mal latency distinguishes V-I from the other groups, the interocular difference in latency differentiates between all three groups.

The relationship between maximal latency and interocular difference in latency is shown in Fig. 3. Each symbol represents one patient, coded with reference to visual findings. The dashed lines indicate the upper limits of normal for maximal latency and interocular difference in latency used as guidelines in this laboratory. They represent two standard deviations above the mean latency and three standard deviations above the mean interocular difference in latency for subjects with normal vision and no neurological complaint.

These guidelines have been selected in view of the known stability of the absolute latency of checkerboard VER, not yet demonstrated for the interocular difference in latency. Patients with clear evidence of optic nerve disease (open circles) are the most readily distinguished from normal subjects by both of these measures. Patients with no visual complaints (triangles) are the most similar to normal subjects on these measures. There are, however, a number of patients with no visual complaints who have long maximal latencies but normal interocular differences in latency. Patients with normal or borderline maximal latencies tend to have no visual complaints unless the interocular difference is greater than normal. Patients having different visual findings are not distributed randomly with regard to normality on either of these measures (Table 3 ). These findings are significant.

To assess the relationship between VER waveform and visual findings, waveforms were classified into three categories: $\mathrm{A}-\mathrm{a}$ single prominent positive peak; $\mathrm{B}$-a double positive peak; $\mathrm{C}-$ no definite peak at the expected latency (distorted response). There were no specific amplitude or 


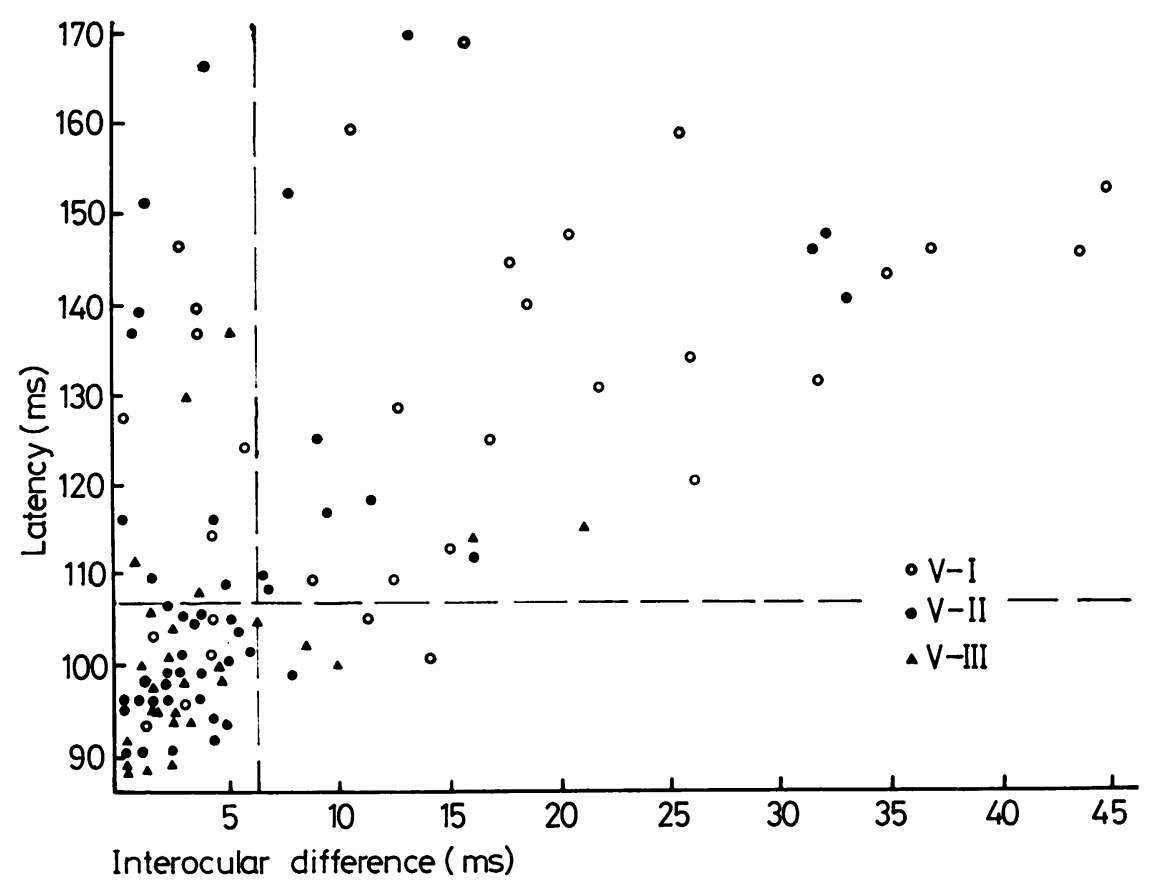

Fig. 3 Distribution of absolute VER latency with respect to interocular difference in latency for patients with different visual findings. Dashed line indicates the upper limit of normal for each parameter.

Table 3 A Distribution of abnormal latencies in $V E R$ according to visual findings

\begin{tabular}{llllll}
\hline & & \multicolumn{3}{l}{ Group } & \\
\cline { 3 - 5 } Latency & & $V-I$ & $V-I I$ & $V-I I I$ & Total \\
\hline Abnormal & Expected & 16 & 19 & 14 & 49 \\
Normal & Observed & 28 & 14 & 7 & 55 \\
& Expected & 19 & 22 & 14 & 51 \\
\hline Observed & 7 & 27 & 21 & \\
\hline Total & & 35 & 41 & 28 & $\mathrm{~N}=104$ \\
& $x^{2}=35.28$ & $\mathrm{df}=2$ & $\mathrm{P}<0.001$ & & \\
\hline
\end{tabular}

B Distribution of abnormal interocular differences in latency according to visual findings

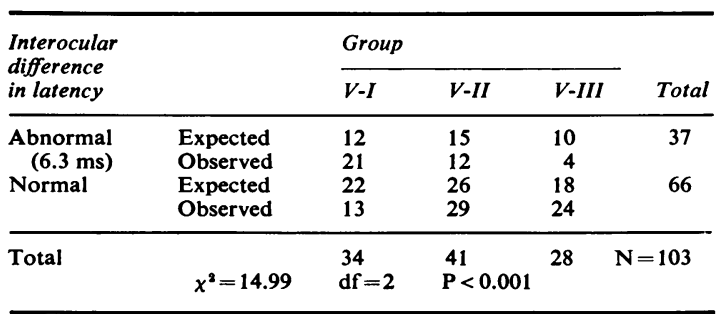

latency criteria. These are the three categories into which evoked responses obtained in our laboratory seem to fall most naturally. Despite the lack of more explicit criteria, waveforms were assigned to these categories separately by two raters with a correlation of 0.95 . The distribution of these waveforms with respect to visual findings is shown in Table 4. Two patients were omitted from the table because of widely divergent ratings.

The waveforms were significantly associated with visual findings. Patients with no visual complaints (V-III) had type A waveforms more frequently than expected by chance. Patients with definite optic nerve involvement (V-I) had a disproportionately small number of type A waveforms and large number of type $\mathrm{C}$ waveforms.

Table 4 Distribution of waveforms of VER in patients according to visual findings

\begin{tabular}{|c|c|c|c|c|c|}
\hline \multicolumn{2}{|l|}{ Group } & $\boldsymbol{A}$ & $B$ & $C$ & Total \\
\hline V-I & $\begin{array}{l}\text { Expected } \\
\text { Observed }\end{array}$ & $\begin{array}{r}13 \\
6\end{array}$ & $\begin{array}{l}15 \\
18\end{array}$ & $\begin{array}{r}6 \\
10\end{array}$ & 34 \\
\hline V-II & $\begin{array}{l}\text { Expected } \\
\text { Observed }\end{array}$ & $\begin{array}{l}15 \\
13\end{array}$ & $\begin{array}{l}18 \\
21\end{array}$ & $\begin{array}{l}7 \\
6\end{array}$ & 40 \\
\hline V-III & $\begin{array}{l}\text { Expected } \\
\text { Observed }\end{array}$ & $\begin{array}{l}11 \\
20\end{array}$ & $\begin{array}{r}13 \\
7\end{array}$ & $\begin{array}{l}4 \\
1\end{array}$ & 28 \\
\hline Total & $x^{2}=22.18$ & $\begin{array}{l}39 \\
\mathrm{df}=4\end{array}$ & $\begin{array}{l}46 \\
P<\end{array}$ & 17 & $=102$ \\
\hline
\end{tabular}




\section{Discussion}

These studies provide evidence of the sensitivity of the visual evoked response to changes in visual function associated with multiple sclerosis. Patients with clinically demonstrable optic nerve lesions are most readily detected by alterations in response latency. The evoked responses of these patients are distinguishable from the responses of patients with visual blurring or diplopia and from patients with no visual complaints on the basis of absolute latency.

On the other hand, patients with visual blurring or diplopia can be distinguished from patients with no visual complaints on the basis of increased interocular difference in latency and not on the basis of absolute latency itself. In fact, patients with substantially prolonged latency from both eyes may be asymptomatic while those with asymmetrically prolonged latencies rarely are.

It appears that asymmetry in conduction from the two eyes to the cortex has functional consequences clinically distinguishable from the effects of symmetrical involvement. Presumably the generation of a clear, unitary image depends on the relative time of arrival of impulses from the two eyes rather than on the absolute time of arrival. Heron et al. (1974) used this principle in showing that a delay in the onset of an increment in light intensity for one eye relative to its fellow could be adjusted to infer the perception of simultaneous onset. The delay was much greater than normal in patients who had experienced a bout of unilateral retrobulbar neuritis. Regan et al. (1976) have extended these observations in measuring both interocular and transretinal delay (suggesting patchy slowing of conduction from two different points on one retina in some multiple sclerosis patients). These delays corresponded to the interocular difference in latency in the steady state responses evoked by pattern stimulation, but not by flicker. Patients with prolonged perceptual delays and interocular differences in evoked response latency were more likely to have visual symptoms.

Our findings, together with the studies mentioned above, indicate that in binocular vision there must be a temporal disparity, comparable to the spatial disparity known as Panum's area, beyond which a unified image cannot be achieved. Beyond this point the visual symptoms of blurring or diplopia may occur. This does not rule out the occurrence of diplopia associated with ocular motor disorders, but rather suggests that similar symptoms may be produced by excessive temporal or spatial disparity in the arrival of impulses at the cortex. However, we are not aware of any report showing delayed conduction associated with misalignment of the eyes.

The alterations in VER waveforms are similar to those observed by Feinsod and Hoyt (1975), but our findings provide clear evidence that abnormal waveforms are correlated with the appearance of clinical symptoms. Such symptoms may be the manifestation of inhomogeneous involvement of optic fibres.

Thus, all of the VER parameters examinedabsolute latency, interocular difference in latency, waveform-are sensitive to involvement of the visual pathways. Nonetheless, it has been demonstrated clearly that in patients who have recovered clinically from optic neuritis there is a lasting deficit in conduction speed, although VER amplitude may have returned to normal (Halliday et al., 1972; Wildberger et al., 1976). These observations suggest that recovery of function (disappearance of symptoms) may be due not only to improvement in conduction in the retina and optic nerves but to a reorganisation of central processing to make the most of incoming information.

\section{References}

Asselman, P., Chadwick, D. W., and Marsden, C. D. (1975). Visual evoked responses in the diagnosis and management of patients suspected of multiple sclerosis. Brain, 98, 261-282.

Behrman, J., Nissim, S., and Arden, G. B. (1972). A clinical method for obtaining pattern visual evoked responses. Advances in Experimental Medicine and Biology, 24, 199-206.

Ciganek, L. (1969). Variability of the human visual evoked potential: normative data. Electroencephalography and Clinical Neurophysiology, 27, 25-42.

Feinsod, M., and Hoyt, W. F. (1975). Subclinical optic neuropathy in multiple sclerosis: how early VER components reflect axon loss and conduction defects in optic pathways. Journal of Neurology, Neurosurgery, and Psychiatry, 38, 1109-1114.

Halliday, A. M., and Michael, W. F. (1970). Changes in pattern-evoked responses in man associated with the vertical and horizontal meridians of the visual field. Journal of Physiology, 208, 449-513.

Halliday, A. M., McDonald, W. I., and Mushin, J. (1972). Delayed visual evoked responses in optic neuritis. Lancet, 1, 982-985.

Halliday, A. M., McDonald, W. I., and Mushin, J. (1973). Visual evoked responses in the diagnosis of multiple sclerosis. British Medical Journal, 4, 661664.

Heron, J. R., Regan, D., and Milner, B. A. (1974). Delay in visual perception in unilateral optic atrophy after retrobulbar neuritis. Brain, 97, 6978.

Kopell, B. S., Wittner, W. K., and Warrick, G. L. 
(1969). The effects of stimulus differences, light intensity, and selective attention on the amplitude of the visual averaged evoked potential in man. Electroencephalography and Clinical Neurophysiology, 26, 619-622.

Lumsden, C. E. (1970). The neuropathology of multiple sclerosis. In Handbook of Clinical Neurology. Edited by P. J. Vinken and G. W. Bruyn, Vol. 9, pp. 217-309. North-Holland: Amsterdam.

McAlpine, D., Lumsden, C., and Acheson, E. (1972). Multiple Sclerosis: A Reappraisal. Williams and Wilkins: Baltimore.

McDonald, W. I. (1974). Pathophysiology in multiple sclerosis. Brain, 97, 179-196.

Regan, D., Milner, B. A., and Heron, J. R. (1976). Delayed visual perception and delayed visual evoked potentials in the spinal form of multiple sclerosis and in retrobulbar neuritis. Brain, 99, 43-63.

Richey, E. T., Kooi, K. A., and Tourtellotte, W. W. (1971). Visually evoked responses in multiple sclerosis. Journal of Neurology, Neurosurgery, and Psychiatry, 34, 275-280.

Wildberger, H., van Lith, G., and Mak, G. (1976). Comparative study of flash and pattern evoked VECPs in optic neuritis. Ophthalmic Research, $8,179-185$. 